\title{
MANET Protocols Performance in Group-based Networks
}

\author{
Jaime Lloret ${ }^{1}$, Miguel García ${ }^{2}$, Fernando Boronat ${ }^{3}$ and Jesus Tomás ${ }^{4}$ \\ Department of Communications, Polytechnic University of Valencia \\ Camino Vera, s/n, Valencia 46022, Spain \\ 1jlloret@dcom.upv.es, ${ }^{2}$ migarpi@ @osgrado.upv.es, ${ }^{3}$ fboronat@dcom.upv.es, ${ }^{4}$ jtomas@upv.es
}

\begin{abstract}
Many routing protocols for ad-hoc and sensor networks have been designed, but none of them are based on groups. It is known that grouping nodes gives better performance to the group and to the whole system, thereby avoiding unnecessary message forwarding and additional overhead. In this paper we show the efficiency of the MANET routing protocols when the nodes are arranged in groups. In order to do it, first, we study the advantages of grouping nodes in each individual protocol for both fixed and mobile networks (nodes with random mobile behaviour). Then, the routing protocols will be compared to analyse which one has the best performance when it is used in a group-based network. This paper shows that group-based systems applied to ad-hoc networks provides better performance than when they are not arranged in groups.
\end{abstract}

\section{Introduction}

MANET networks [1] are a type of ad-hoc networks much more studied and mature than Wireless Sensor Networks (WSN) [2]. Independently of the medium access method used [3], in the recent years many routing protocols have been developed for MANET networks [4] [5]. The nodes' mobility, the lack of stability of the topology, the lack of a pre-established organization and performing of the wireless communications are reasons for not using the routing protocols developed for fixed networks. There are standardized routing protocols for MANET networks used by different fixed or mobile devices.

Depending on the information type exchanged by the nodes and on the frequency they do it, the routing protocols in ad-hoc networks are divided into three types: proactives, reactives and hybrids. The proactive protocols update the routing tables of all the nodes periodically, even though no information is being exchanged. When a topology change occurs, the routing table is updated and the 
routing protocol finds the optimum route to forward the information. A periodical control protocol message exchange allows this, but consumes bandwidth and energy (batteries). The reactive protocols only maintain routing routes in their tables when a node has to communicate with another node in the network. With these protocols, when a communication starts, as the right route is unknown, a route discovering message is sent. When the response is received, the route is included in the routing tables and the communication is now possible. The main disadvantage of these protocols is the latency at the beginning of the communications (route discovery time) but they improve the network and energy resources use. Inside this kind of protocols, we can find the source-based protocols and hop-by-hop protocols. Finally, the hybrid protocols are a combination of the other two types, taking the advantages of both types. These protocols divide ad-hoc networks into different zones, and then near nodes use proactive routing meanwhile far nodes use reactive routing.

Current IETF standardized protocols are AODV (Ad-Hoc On-demand Distance Vector) [6], DSR (Dynamic Source Routing Protocol) [7] and OLSR (Optimized Link State Routing Protocol) [8]. So, we are going to analyze and study their performance in this paper.

AODV is a routing protocol for Mobile ad-hoc networks and is a reactive protocol. It has a minimalist behaviour because it hardly overloads the ad-hoc network and needs very few memory comparing with other protocols. It works over IP protocol.

DSR is a reactive protocol developed specifically for ad-hoc networks. It only sends information when it is required, saving bandwidth, energy and battery. The protocol has two mechanisms: route discovering and route maintenance. It also includes a mechanism to avoid loops. It is compatible with IPv6 (IP, version 6). It has the following disadvantages: the excessive initial latency while discovering the route; and, as a source-based protocol, the size of the packet header increases each time it goes through a node, affecting to the bandwidth consumption.

OLSR is a proactive protocol in which each node knows permanently the network state and the number, availability and addresses of the nodes. This performs a faster routing protocol. OLSR optimizes the time of response when a change is detected in the network, by reducing the period time of the control messages transmission. As routes for all the destinations are maintained, it has a quite good performance in networks with random and sporadic traffic in large groups of nodes. As disadvantages we can point the followings: the nodes require more memory resources and it overloads the network with routing control packets. OLSR was developed to work, in an independently way, with other protocols, bringing versatility to use it in any scenario. The most important key in this protocol is MPR (Multipoint Relay) node, it optimizes the number of control messages in the network.

There are several works published that compare MANET routing protocols [9] [10] [11] [12], but none of them have compared MANET routing protocols from the group-based network point of view. In order to do our comparison, we have 
used the version Modeler of OPNET simulator [13]. Our goal is to evaluate the performance of three MANET routing protocols from the point of view of some parameters such as: network load when the network is stable, network load when there are topology changes, convergence time, number of updates, correct sent/received packets, wrong sent/received packets, etc.

This paper is structured as follows. Section 2 explains group-based networks benefits, describes some group-based protocols and explains where group-based protocols could be implemented. Our simulations, analysis and comparison are shown in section 3. Finally, section 4 gives the conclusions and future works.

\section{Group-based networks benefits and related works}

A group is referred as a small number of interdependent nodes that interact in order to share resources or computation time and produce joint results. In a physical group-based architecture neighboring groups could be connected if border nodes from different groups are close. A group-based network is capable of having different types of topologies and protocols inside every group. There are some works in the literature where nodes are divided into groups and links between nodes from different groups are taken into account, but all of them have been developed to solve specific issues and none of them for MANET networks. Rhubarb system [14] organizes nodes in a virtual network, allowing connections across firewalls/NAT, and efficient broadcasting. Rhubarb system has only one coordinator per group and coordinators could be grouped in groups in a hierarchy. A Peer-toPeer Based Multimedia Distribution Service was presented in [15]. Authors propose a topology-aware overlay in which nearby hosts or peers self-organize into application groups. End hosts within the same group have similar network conditions and can easily collaborate with each other to achieve QoS awareness. There are some architectures, such as [16] and [17], were nodes are structured hierarchically and parts of the tree are grouped into groups. In some cases, some nodes have connections with nodes from other groups although they are in different layers of the tree, but the information has to be routed through the hierarchy.

Finally, we want to emphasize that the cluster-based networks are a subset of the group-based networks, because every cluster could be considered as a group. A cluster can be made up of a Cluster Head node, Cluster Gateways and Cluster Members ([18] [19]). The Cluster Head node is the parent node of the cluster, which manages and checks the status of the links in the cluster, and routes the information to the right clusters. The rest of the nodes in a cluster are all cluster members and don't use to have inter-cluster links. The size of the cluster is usually about 1 or 2 hops from the Cluster Head node while a group could be as large as we want. All the clusters have the same rules, however, a group-based network is capable of having any type of topology inside the group, not only clusters. 
Group-based networks provide some benefits for the whole network such as:

- Spreads the work to the network in groups giving more flexibly, efficiently and lower delays.

- Content availability will increase because it could be replicated to other groups.

- Anyone could search and download from every group using only one service.

- It provides fault tolerance. Other groups could carry out tasks from a failed one.

- It is scalable because a new group could be added to the system easily.

- Network measurements could be taken from any group.

Group-based networks have many application areas. They could be used when it is wanted to setup a network where groups could appear and join the network anytime or by networks have to be split into smaller zones to support a large number of nodes, that is, any system where the devices are grouped and there must be connections between groups. The main goal in a group-based topology is the network protocol and the group management, that is, the design of an efficient algorithm for a new node to find its nearest (or the best) group to join in. The performance of the network highly depends on the efficiency of the nearby group locating process and on the interaction between neighboring groups.

The following list gives several group-based ad-hoc networks application areas: 1. Let us suppose a job where all human resources need to be split into groups to achieve a purpose (such as fire fighter squads for putting out the fire). Now, let's suppose that all people involved in that activity need a device that has to be connected with other devices in the same group to receive information from the members within the group, and closer groups have to be connected to coordinate their efforts. Currently coordination between groups is done through a wireless connection to the command center or using satellite communications. But, some times neither of those solutions can be used because a free of obstacles line of sight is needed, because there are too many wall looses or because more gain or power is needed to reach the destination.

2. Groups could also be established because of geographical locations or unevenness. It happens in rural and agricultural environments. A group-based topology in this kind of environment could be useful to detect plagues or fire and to propagate an alarm to neighbor lands. It will provide easier management and control for detecting fires and plagues as well as allowing scalability.

3. It could be used in any kind of system in which an event or alarm is based on what is happening in a specific zone, but conditioned to the events that are happening in neighbor zones. One example is a group-based system to measure the environmental impact of a place. It could be better measured if the measurements are taken from different groups of nodes, but those groups of nodes have to be connected in order to estimate the whole environmental impact.

4. Group-based virtual games. There are many games where the players are grouped virtually in order to perform a specific task. Interactions between groups in virtual reality should be given by interactions between players from different groups to exchange their knowledge. 


\section{Group-based ad-hoc networks analysis}

This section describes how are the test bench and the traffic used for simulations, in the regular and the group-based topologies, to take measurements.

\subsection{Test bench}

We used the same test-bench for all the evaluated protocols using OPNET Modeler [13]. We varied the number of nodes and the coverage area in an open environment. The nodes in the topology have the characteristics of an ad-hoc node (40 MHz processor, $512 \mathrm{~KB}$ memory card, radio channel of $11 \mathrm{Mbps}$ and $2.4 \mathrm{GHz}$ as the work frequency). The MAC protocol was CSMA/CA. We chose nodes with a 50 meters maximum coverage radius. This is a conservative value, so the simulations presented in this work give us an adequate view for the worst case.

We simulated 4 scenarios for each protocol: the first one with fixed nodes; the second one with mobile nodes and failures; the third one with grouped nodes; and, the fourth one with grouped mobile nodes and failures. For each topology, we simulated for 100 and 250 nodes, to observe the system scalability.

Instead of a standard structure we chose a random topology where the nodes are mobile and change their position constantly. The groups are created by physical coverage area. When a node moves into a new coverage area, it belongs to the new group. 100 nodes topology has a $750 \times 750 \mathrm{~m}^{2}$ area (density ! $0.18 \cdot 10^{-3}$ nodes $/ \mathrm{m}^{2}$ ) and 250 nodes topology has a $1 \mathrm{Km}^{2}$ area (density ! $0.25 \cdot 10^{-3}$ nodes $\left./ \mathrm{m}^{2}\right)$. We forced failures at $\mathrm{t}=200 \mathrm{sec}$., $\mathrm{t}=400 \mathrm{sec}$. and $\mathrm{t}=1200 \mathrm{sec}$. in each network, with a recovering process of $300 \mathrm{sec}$., to take measurements when the physical topology changes. We are going to study how several network-level protocols perform when failures and recoveries happen in this kind of networks.

We created 6 groups for the 100 nodes topology, each group covers a circular area of 150 meter radius. They were arranged to cover the whole area. There were approximately 16 or 17 nodes, in each group in the initial process. The number of nodes in each group varied because of the node's random mobility, so in one instant a node could belong to a group and in another instant to another one. We created 12 groups with 15 or 16 nodes per group for the 250 nodes topology. The group's coverage areas were similar for both areas. The routing protocol used inside the group will be the same as the one used between groups.

The traffic load used for the simulations is MANET traffic generated by OPNET. We inject this traffic 100 seconds after the beginning. The traffic follows a Poisson distribution (for the arrivals) with a mean time between arrivals of 30 seconds. The packet size follows an exponential distribution with a mean value of 1024 bits. The injected traffic has a random destination address, to obtain a simulation independent of the traffic direction. We have simulated the four scenarios for DSR, AODV and OLSR protocols. 


\subsection{Simulation results and analysis for DSR protocol}

In figures 1 and 2 we can see the MAC level mean delay experimented by traffic using CSMA/CA. In figure 1, the group-based topology has a mean delay of 250 $\mu \mathrm{s}$ independently of the number of nodes in the network. The regular topology converges around $1.1 \mathrm{~ms}$. The difference between both cases is about $850 \mu \mathrm{s}$; therefore the MAC level mean delay decreases a percentage of $23 \%$ in both cases. In figure 2 , we can see that the delays are lower. It is mainly because of the network mobility. In this case, we appreciate that there are differences between 100node and 250-node topologies. In group-based topologies the MAC level mean delay is around $100 \mu \mathrm{s}$ for both topologies, so they converge faster.

When we study the network throughput consumed (figures 3 and 4), we observe that group-based topologies give a much lower value than the one obtained in regular topologies. For the 100-node topology (figure 3), the mean throughput varies from $225 \mathrm{Kbits} / \mathrm{s}$ to $100 \mathrm{Kbits} / \mathrm{s}$ in the group-based topology (a 56\% improvement). In the 250-node topology we obtain $460 \mathrm{Kbits} / \mathrm{s}$ of throughput for the regular topology and $190 \mathrm{Kbits} / \mathrm{s}$ of throughput for the group-based one (a 59\% improvement). Moreover, when we compare figures 3 and 4, we can conclude that the throughput in group-based topologies has a very low variation regarding a fixed or mobile scenario. The obtained improvement is quite important. We can see in figure 4 that, after 1200 seconds, the obtained throughput in 250-node topology is similar to the obtained throughput in the 100-node regular topology.

Observing figures 5 and 6, we conclude that the MANET traffic load through the network is lower for group-based topologies. In both figures we can see that when the number of nodes increases the traffic decreases. This is due to the existence of more nodes working as routers and therefore the probability of a packet to reach the destination is higher. The 100-node group-based topology (figure 5) gives a $77 \%$ improvement regarding the regular 100-node topology, but the improvement decreases when the number of nodes increases (in 250-node cases the improvement is about $60 \%$ ). This behavior varies when the topology has mobility, errors and failures (figure 6). In this case, the 100 node group-based topology also has improvement (around $77 \%$ ) regarding to the 100 -node regular one. This improvement is higher $(80 \%)$ in 250 -node topologies.

We have also compared the routing traffic sent (figures 7 and 8). In figure 7 we observe that the traffic is quite stable due to the characteristics of the network, because it is a fixed network without errors and failures. The traffic sent in 250-node topology is around $225 \mathrm{Kbits} / \mathrm{s}$, but when we group the nodes this traffic decreases to $100 \mathrm{Kbits} / \mathrm{s}$ (a $60 \%$ improvement). The value obtained in a 100-node topology is also improved when we group the nodes $(50 \mathrm{Kbits} / \mathrm{s}$, therefore a $50 \%$ improvement). In figure 8 we observe a similar behavior. In this case we conclude that when there are errors and failures in the network (interval from 600 to $800 \mathrm{sec}$ onds and around 1200 seconds) the traffic fluctuates and is less stable. We appreciate the instability is much lower in group-based topologies. 


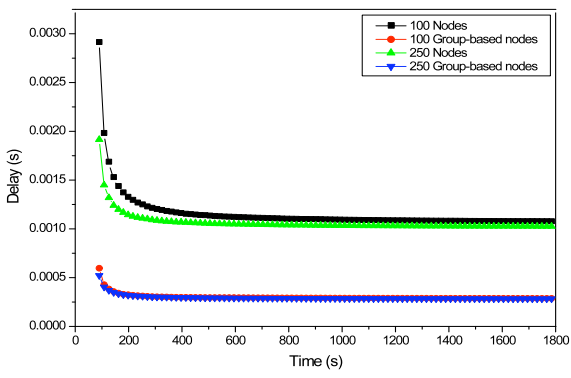

Fig. 1 DSR mean delay in fixed topologies.

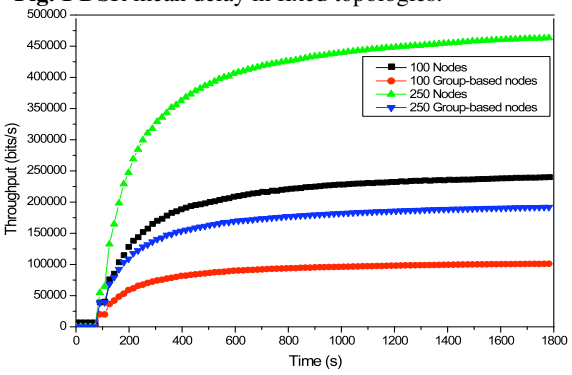

Fig. 3 DSR mean throughput in fixed topologies.

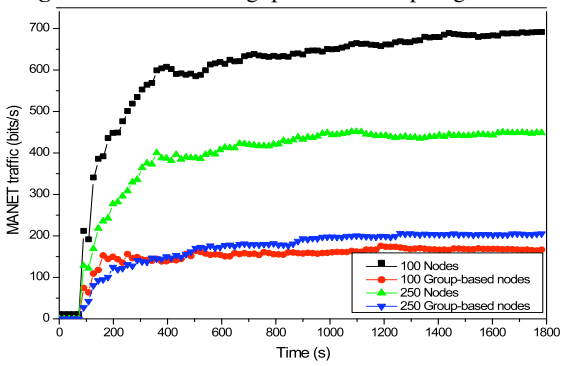

Fig. 5 DSR mean MANET traffic in fixed topologies.

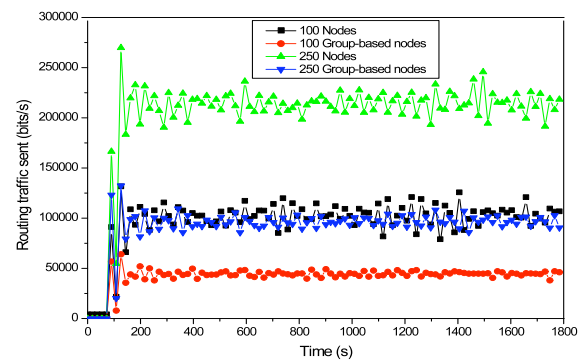

Fig. 7 DSR routing traffic in fixed topologies.

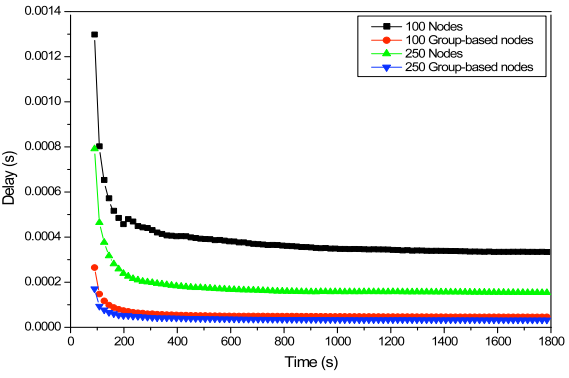

Fig. 2 DSR mean delay in mobile topologies.

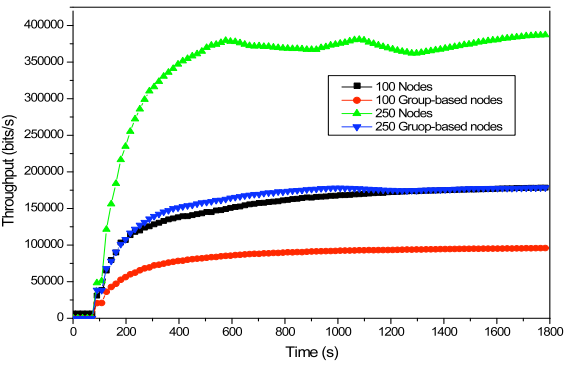

Fig. 4 DSR mean throughput in mobile topologies.

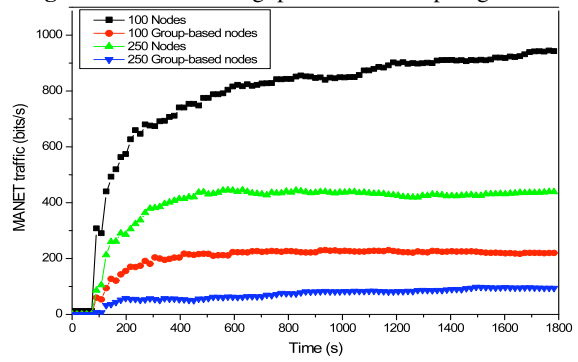

Fig. 6 DSR mean MANET traffic in mobile topologies.

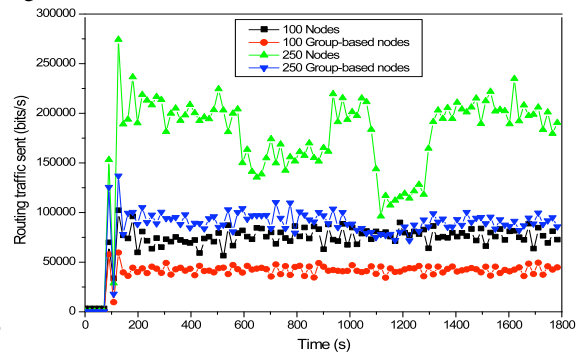

Fig. 8 DSR routing traffic mobile topologies. 


\subsection{Simulation results and analysis for $A O D V$ protocol}

Figures 9 and 10 show the MAC level mean delay for AODV protocol simulations. We can observe that the delay has no strong dependence of both the topology type and the number of nodes. For 100 and 200-node topologies we obtain a delay stabilized around $1 \mathrm{~ms}$, but for group-based topologies this value is $300 \mu \mathrm{s}$. Therefore it decreases a percentage of $70 \%$ in both cases.

Figure 11 shows the mean throughput for fixed topologies. The 100-node scenario gives a $200 \mathrm{Kbits} / \mathrm{s}$ mean value, but a value of $120 \mathrm{Kbits} / \mathrm{s}$ is obtained for the group-based scenario (a $40 \%$ improvement). In the 250 -node case, we obtain mean values of $425 \mathrm{Kbits} / \mathrm{s}$ for the fixed scenario and of $225 \mathrm{Kbits} / \mathrm{s}$ for the groupbased scenario (a 47\% improvement). Figure 12 shows the results for mobile topologies with errors and failures. The improvement, obtained by grouping nodes, decreases in the 100-node case (37\%), but it doesn't vary in the 250-node cases.

Figures 13 and 14 show the evolution of the MANET traffic for different scenarios. In figure 13, the traffic of the 100-node fixed topology has a mean value of around $600 \mathrm{bits} / \mathrm{s}$, but it decreases to $180 \mathrm{bits} / \mathrm{s}$ for the 100-node group-based scenario, giving a $70 \%$ of improvement. In the 250-node topologies, it has varied from $480 \mathrm{Kbits} / \mathrm{s}$ (fixed) to $50 \mathrm{Kbits} / \mathrm{s}$ (group-based), obtaining a 90\% improvement. In figure 14 we appreciate the improvement (not too relevant as in above case) and we can see the fast convergence of group-based topologies for mobile topologies with errors and failures. In the 100-node case the traffic doesn't converge before 1400 seconds, but it converges in 200 seconds when there are group of nodes. It also happens in the 250-node topologies: the regular topology converges in 600 seconds while the group-based one converges in 180 seconds.

The routing traffic measured in each simulated scenario can be seen in figures 15 and 16 . We observe that the routing traffic is independent of the node mobility. In figure 15 we can see that the routing traffic goes from $200 \mathrm{Kbits} / \mathrm{s}$ for 250-node case to $125 \mathrm{Kbits} / \mathrm{s}$ when there are group of nodes (a $37 \%$ improvement). In the 100 -node cases, it goes from $90 \mathrm{Kbits} / \mathrm{s}$ to $50 \mathrm{Kbits} / \mathrm{s}$ (a $45 \%$ improvement). When there are mobility, errors and failures (see figure 16), in the 250-node topology the values go from $190 \mathrm{Kbits} / \mathrm{s}$ to $110 \mathrm{Kbits} / \mathrm{s}$ in the group-based scenario (a $42 \% \mathrm{im}$ provement). We obtained $85 \mathrm{Kbits} / \mathrm{s}$ for the regular 100-node topology and 50 Kbits/s for the group-based one (a $41 \%$ improvement).

\subsection{Simulation results and analysis for OLSR protocol}

Figure 17 shows the MAC level mean delay in fixed topologies. In the 250-node regular topology we obtained a value around $920 \mu \mathrm{s}$ and a value around $250 \mu \mathrm{s}$ in the group-based one (a 73\% improvement). With 100 nodes there is no a significant improvement in the group-based topology (both values are around $260 \mu \mathrm{s}$ ). 

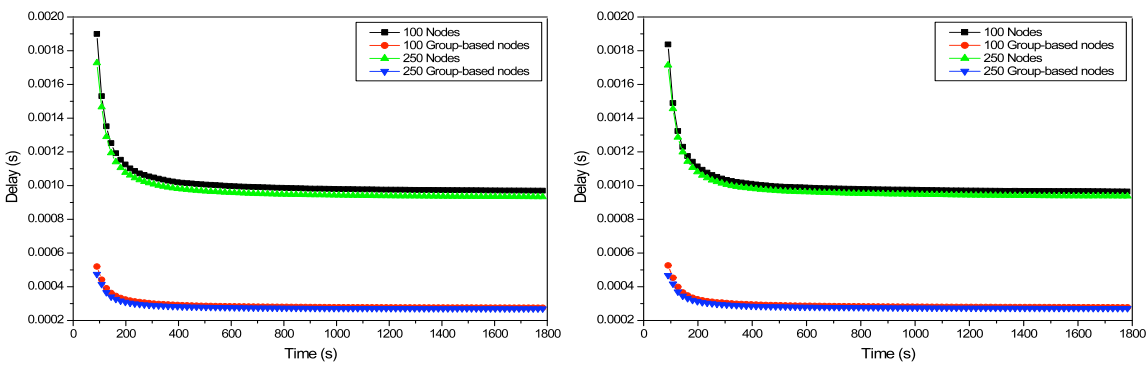

Fig. 9 AODV mean delay in fixed topologies

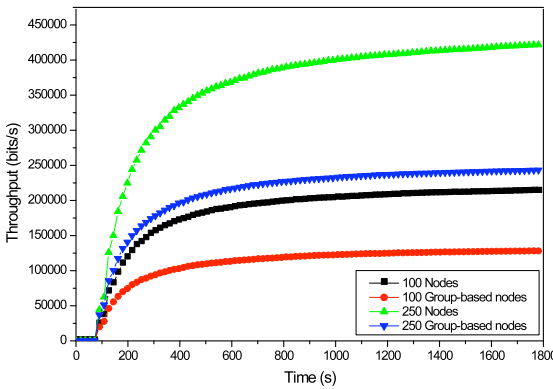

Fig. 10 AODV mean delay in mobile topologies.

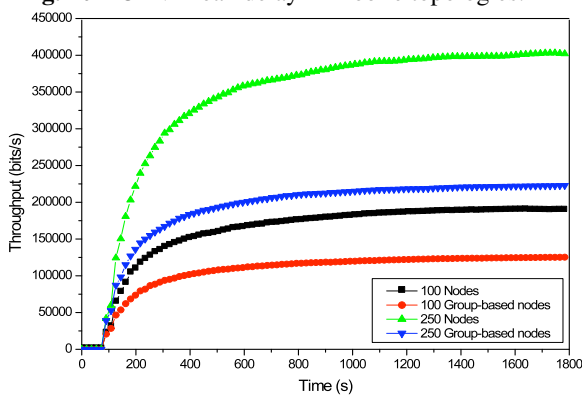

Fig. 11 AODV mean throughput consumed in fixed topologies.

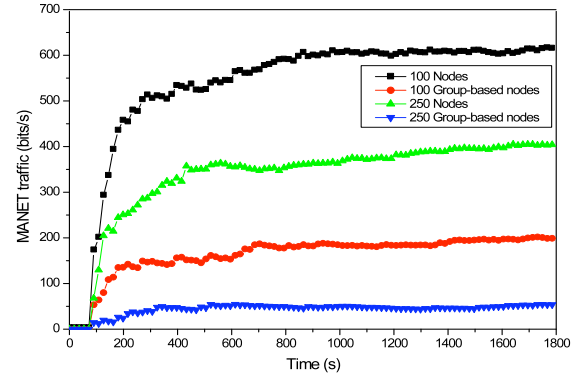

Fig. 12 AODV mean throughput consumed in mobile topologies.

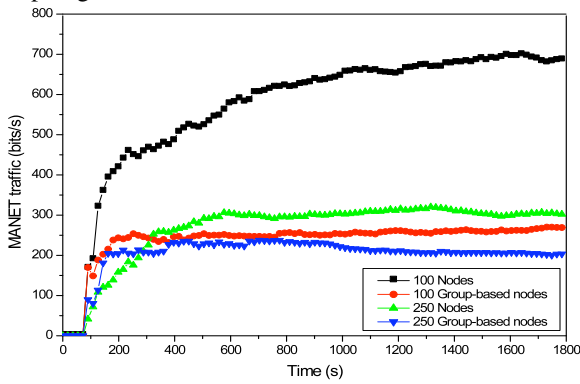

Fig. 13 AODV mean MANET traffic in fixed topologies.

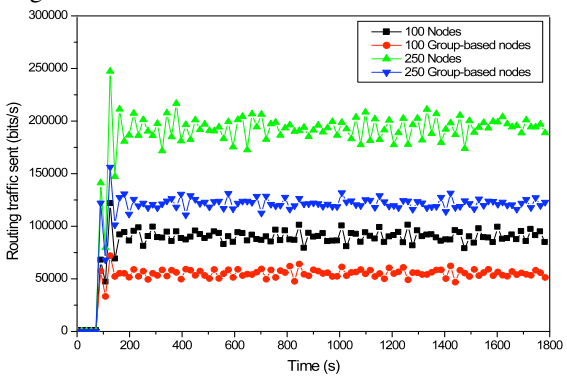

Fig. 14 AODV mean MANET traffic in mobile topologies.

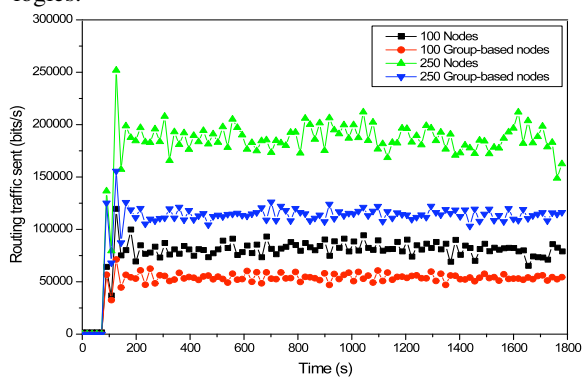

Fig. 15 AODV routing traffic in fixed topologies. Fig. 16 AODV routing traffic in mobile topologies. 
Figure 18 shows the cases when there is mobility, errors and failures. In 100node topologies, the regular topology has a mean value of $268 \mu \mathrm{s}$ and the groupbased scenario has $262 \mu \mathrm{s}$. In 250-node case, the improvement is not so good (from $262 \mu$ s to $260 \mu$ s).

The mean throughput consumed in fixed topologies can be observed in figure 19. In the scenarios with 250 nodes we obtained throughputs of $550 \mathrm{Kbits} / \mathrm{s}$ and $250 \mathrm{Kbits} / \mathrm{s}$ (group-based has 54\% improvement). In 100-node regular topology the throughput is $325 \mathrm{Kbits} / \mathrm{s}$ and $125 \mathrm{Kbits} / \mathrm{s}$ (group-based has $61 \%$ improvement). When we consider mobility (figure 20) the consumed throughput is not so stable as in above case but, we can observe that the improvements are quite similar. In the case of 250 nodes we obtain a $52 \%$ improvement in group-based scenario; in the case of 100 nodes the improvement reaches the $60 \%$.

When we analyze the mean MANET traffic through the network, we obtain the results shown in figure 21 and 22. In figure 21, the mean traffic in the 100-node topology was $700 \mathrm{bits} / \mathrm{s}$ for the regular topology and $180 \mathrm{bits} / \mathrm{s}$ for the group-based topology, obtaining a $75 \%$ improvement. In 250-node regular scenario, we obtained around $450 \mathrm{bits} / \mathrm{s}$, but a value of $220 \mathrm{bits} / \mathrm{s}$ when we group nodes. There is around $51 \%$ improvement. Figure 22 shows the value of MANET traffic in mobile topologies with errors and failures. We appreciate that regular topologies have higher convergence time than the group-based topologies. In this case, the 100node topology did not converge in the simulated interval. In the 250-node topology, the network gets stability after 1200 seconds. The improvement of the 250 node topologies is around $51 \%$. The mean value of the MANET traffic obtained in the regular 100-node topology is around $900 \mathrm{bits} / \mathrm{s}$ and $215 \mathrm{bits} / \mathrm{s}$ in the groupbased topology, giving a $76 \%$ improvement.

Finally, we have analyzed the mean routing traffic sent in fixed and mobile topologies (figures 23 and 24). The routing traffic sent in the 100-node fixed topology was around $60 \mathrm{Kbits} / \mathrm{s}$, while in the group-based topology was $28 \mathrm{Kbits} / \mathrm{s}$, with a $53 \%$ improvement. In the 250-node case, we appreciate that this traffic was higher than $110 \mathrm{Kbits} / \mathrm{s}$, but only higher than $60 \mathrm{Kbits} / \mathrm{s}$ for the group-based scenario, with a $45 \%$ improvement (figure 23 ). Figure 24 shows the results of a network with mobility, errors and failures. The routing traffic is quite dependent of the failures in the network. In both 100-node and 250-node scenarios there are some fluctuations due to the inherent characteristics of the network that are minimized grouping the nodes. Improvements of $45 \%$ and $53 \%$ are obtained in 100node and 250-node scenarios, respectively. So, we can emphasize the good scalability of the group-based topologies.

\section{Conclusions}

In this work we have shown the benefits of using a group-based topology in adhoc networks and we have shown several examples where they can be used. 

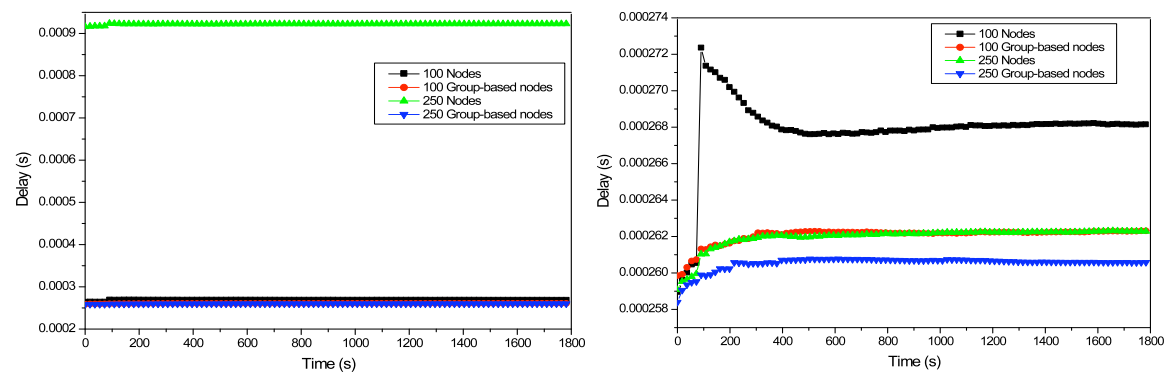

Fig. 17 OLSR mean delay in fixed topologies.

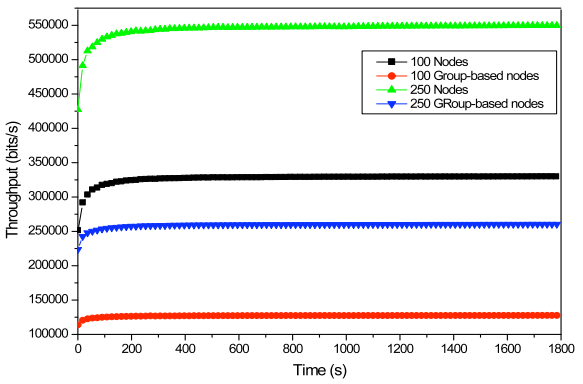

Fig. 18 OLSR mean delay in mobile topologies.

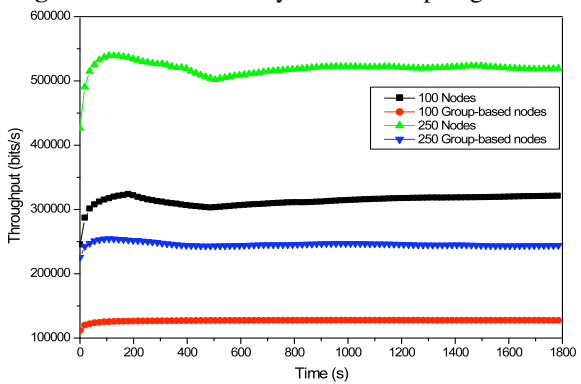

Fig. 19 OLSR mean throughput consumed in fixed topologies.

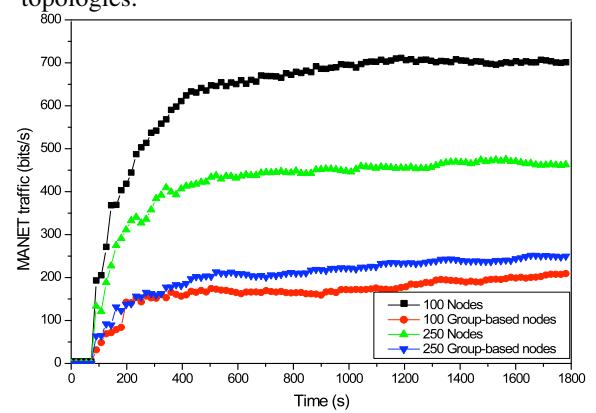

Fig. 20 OLSR mean throughput consumed in mobile topologies.

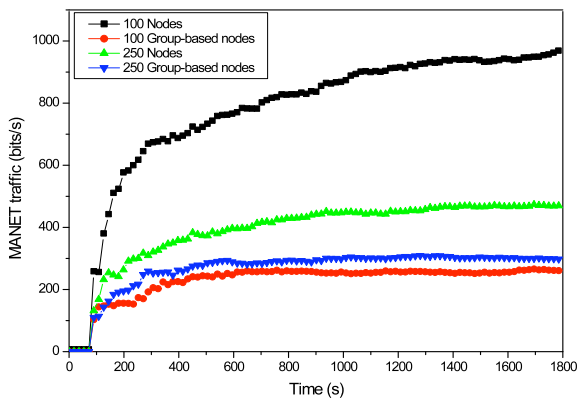

Fig. 21 OLSR mean MANET traffic in fixed topologies.

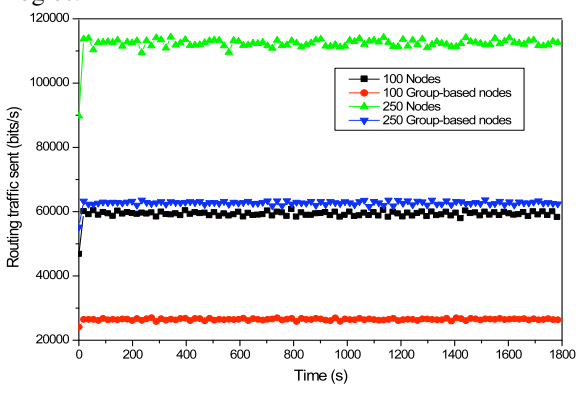

Fig. 22 OLSR mean MANET traffic in mobile topologies.

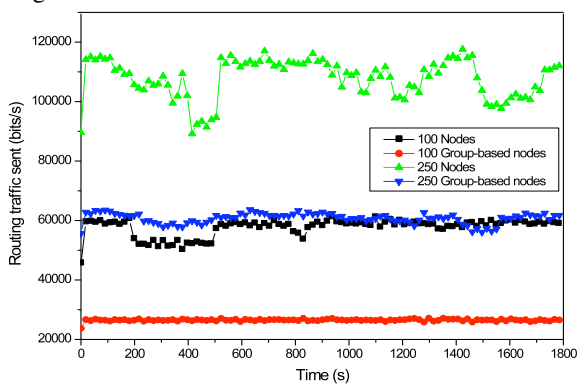

Fig. 23 OLSR routing traffic in fixed topologies.

Fig. 24 OLSR routing traffic in mobile topologies. 
We have simulated DSR, AODV and OLSR protocols with and without groups and the results show that group-based topologies give better performance for wireless ad-hoc networks. So, grouping nodes increases the productivity and the performance of the network with low overhead and low extra network traffic. Therefore, good scalability can be achieved in group-based networks. On the other hand, the protocol that gives better results has been OLSR because this protocol introduces less routing traffic and it has behavior more regular.

\section{References}

1. M. Frodigh, P. Johansson, P. Larsson. "Wireless ad hoc networking. The art of networking without a network". Ericsson Review, No. 4, 2000.

2. I. F. Akyildiz, W. Su, Y. Sankarasubramaniam and E. Cayirci. "A survey on sensor networks". Communications Magazine, IEEE. Volume 40, Issue 8. Pp. 102- 114. Aug 2002.

3. S. Kumar, V. S. Raghavan, J. Deng. "Medium Access Control protocols for ad hoc wireless networks: A survey". Ad Hoc Networks. Volume 4, Issue 3, Pp. 326-358. May 2006.

4. E. M. Royer and C.-K. Toh, "A Review of Current Routing Protocols for Ad Hoc Mobile Wireless Networks," IEEE Personal Communications, April 1999, pp. 46-55.

5. R. Rajaraman, "Topology control and routing in ad hoc networks: a survey". ACM SIGACT News. Volume 33, Issue 2, Pp. 60-73. June 2002.

6. C. Perkins, E. Belding-Royer and S. Das, "Ad Hoc On-Demand Distance Vector (AODV) Routing". RFC 3561. July, 2003.

7. D. Johnson, Y. Hu, D. Maltz. "The Dynamic Source Routing Protocol (DSR) for Mobile Adhoc Networks for IPv4". RFC 4728. February, 2007.

8. T. Clausen and P. Jacquet. "Optimized Link State Routing Protocol". RFC 3626. Oct. 2003.

9. Josh Broch, David A. Maltz, David B. Johnson, Yih-Chun Hu, Jorjeta Jetcheva, "A Performance Comparison of Multi-Hop Wireless Ad Hoc Network Routing Protocols". Proc. of the 4th ACM/IEEE int. conf. on Mobile computing and networking. Pp. 85-97. 1998.

10. P. Johansson, T. Larsson, N. Hedman, B. Mielczarek, and M. Degermark, "Scenario-Based Performance Analysis of Routing Protocols for Mobile Ad Hoc Networks". Proc. of the 5th ACM/IEEE int. conf. on Mobile computing and networking. Pp. 195-206. 1999.

11. C. E. Perkins, E. M. Royer, S. R. Das, M. K. Marina, Performance Comparison of Two OnDemand Routing Protocols for Ad Hoc Networks, IEEE Personal Com. Pp. 16-28. Feb. 2001.

12.X. Hong, K. Xu, M. Gerla. "Scalable routing protocols for mobile ad hoc networks". IEEE Network. Volume 16, Issue 4, Pp. 11-21. Jul/Aug 2002.

13. OPNET Modeler website. At, http://www.opnet.com/solutions/network_rd/modeler.html

14. Wierzbicki, A., Strzelecki, R., Swierczewski, D. and Znojek, M. Rhubarb: a Tool for Developing Scalable and Secure Peer-to-Peer Applications. Second IEEE International Conference on Peer-to-Peer Computing (P2P2002), Linöping, Sweden, 2002.

15. Xiang, Z., Zhang, Q., Zhu, W., Zhang, Z. and Zhang, Y. Peer-to-Peer Based Multimedia Distribution Service, IEEE Transactions on Multimedia 6 (2) (2004).

16. L. Hongjun, L. P. Luo, Z. Zhifeng. A structured hierarchical P2P model based on a rigorous binary tree code algorithm, Future Generation Computer Systems 23 (2). 2007. Pp. 201-208.

17. B. Thallner, H. Moser. Topology control for fault-tolerant communication in highly dynamic wireless networks. $3^{\text {rd }}$ Wrk. on Intelligent Solutions in Embedded Systems. May 2005.

18. M. Jiang, J. Li, Y.C. Tay. "Cluster Based Routing Protocol (CBRP)". Internet-draf, draft-ietfmanet-cbrp-spec-01.txt, National University of Singapore, 14 August 1999.

19. Chiang, C.C., Wu, H.K., Liu, W., Gerla, M., "Routing in Clustered Multihop, Mobile Wireless Networks with Fading Channel", IEEE SICON, Kent Ridge (Singapore). April 1997. 